\title{
Characterization of brain-derived extracellular vesicle lipids in Alzheimer's disease
}

\author{
Huaqi Su ${ }^{1,2}$ | Yepy H. Rustam ${ }^{2}$ | Colin L. Masters ${ }^{1}$ | Enes Makalic ${ }^{3}$ | \\ Catriona A. McLean $^{1} \quad$ | Andrew F. Hill ${ }^{4} \quad$ Kevin J. Barnham ${ }^{1} \quad$ Gavin E. Reid ${ }^{2,5}$ \\ Laura J. Vella ${ }^{1,6}$
}

${ }^{1}$ The Florey Institute of Neuroscience and Mental Health, The University of Melbourne, Parkville,

Victoria, Australia

${ }^{2}$ Department of Biochemistry and Pharmacology, The University of Melbourne, Parkville, Victoria, Australia

${ }^{3}$ Centre for Epidemiology and Biostatistics, Melbourne School of Population and Global Health, The University of Melbourne, Parkville, Victoria, Australia

${ }^{4}$ Department of Biochemistry and Genetics, La Trobe Institute for Molecular Science, La Trobe

University, Bundoora, Victoria, Australia

${ }^{5}$ School of Chemistry, Bio21 Molecular Science and Biotechnology Institute, The University of Melbourne, Parkville, Victoria, Australia

${ }^{6}$ Department of Surgery, The Royal Melbourne Hospital, The University of Melbourne, Parkville, Victoria, Australia

\section{Correspondence}

Gavin E. Reid, Department of Biochemistry and Pharmacology, The University of Melbourne,

Parkville, Victoria, Australia.

Email: gavin.reid@unimelb.edu.au

Laura J. Vella, The Florey Institute of Neuroscience and Mental Health, The University of Melbourne,

Parkville, Victoria, Australia.

Email:ljvella@unimelb.edu.au

Kevin J. Barnham, Gavin E. Reid and Laura J. Vella contributed equally to this study.

Funding information

Australian National Health and Medical Research Council, Grant/Award Numbers: GNT1041413, GNT1002349, 628946; Australian Research Council, Grant/Award Number: LE160100015; Bethlehem Griffiths Research Foundation; Alzheimer's Australia Dementia Research Foundation John Shutes Project Grant; Alzheimer's Association, Grant/Award Number: AARF-18-566256

\begin{abstract}
Lipid dyshomeostasis is associated with the most common form of dementia, Alzheimer's disease (AD). Substantial progress has been made in identifying positron emission tomography and cerebrospinal fluid biomarkers for AD, but they have limited use as front-line diagnostic tools.

Extracellular vesicles (EVs) are released by all cells and contain a subset of their parental cell composition, including lipids. EVs are released from the brain into the periphery, providing a potential source of tissue and disease specific lipid biomarkers. However, the EV lipidome of the central nervous system is currently unknown and the potential of brain-derived EVs (BDEVs) to inform on lipid dyshomeostasis in $\mathrm{AD}$ remains unclear.

The aim of this study was to reveal the lipid composition of BDEVs in human frontal cortex, and to determine whether BDEVs have an altered lipid profile in AD. Using semi-quantitative mass spectrometry, we describe the BDEV lipidome, covering four lipid categories, 17 lipid classes and 692 lipid molecules. BDEVs were enriched in glycerophosphoserine (PS) lipids, a characteristic of small EVs. Here we further report that BDEVs are enriched in ether-containing PS lipids, a finding that further establishes ether lipids as a feature of EVs.

BDEVs in the AD frontal cortex offered improved detection of dysregulated lipids in $\mathrm{AD}$ over global lipid profiling of this brain region. AD BDEVs had significantly altered glycerophospholipid and sphingolipid levels, specifically increased plasmalogen glycerophosphoethanolamine and decreased polyunsaturated fatty acyl containing lipids, and altered amide-linked acyl chain content in sphingomyelin and ceramide lipids relative to CTL. The most prominent alteration was a two-fold decrease in lipid species containing anti-inflammatory/pro-resolving docosahexaenoic acid.

The in-depth lipidome analysis provided in this study highlights the advantage of EVs over more complex tissues for improved detection of dysregulated lipids that may serve as potential biomarkers in the periphery.
\end{abstract}

\section{KEYWORDS}

Alzheimer's disease, brain, exosomes, extracellular vesicles, frontal cortex, glycerophospholipids, lipid biomarkers, lipidome, polyunsaturated fatty acids, sphingolipids, tissue

This is an open access article under the terms of the Creative Commons Attribution License, which permits use, distribution and reproduction in any medium, provided the original work is properly cited.

(c) 2021 The Authors. Journal of Extracellular Vesicles published by Wiley Periodicals, LLC on behalf of the International Society for Extracellular Vesicles 


\section{1 | INTRODUCTION}

Alzheimer's disease (AD) is a neurodegenerative disorder and the most common form of dementia. AD has an extended preclinical window which offers hope in treating the disease, however clinical differentiation and diagnosis remains difficult in the absence of blood-based biomarkers and current therapies are unable to halt disease progression.

Lipids are involved in maintenance of cellular homeostasis and are key components of cellular membranes. They have multiple functional roles, including regulation of energy storage and signal transduction, and also for the segregation of chemical reactions in discrete organelles.

It has previously been reported that lipid metabolism is extensively reprogrammed in $\mathrm{AD}$ with lipid changes previously detected in AD brain, cerebrospinal fluid (CSF) and blood (Han et al., 2011; He et al., 2010; Kosicek \& Hecimovic, 2013; Mielke \& Lyketsos, 2010; Mielke et al., 2010a, 2010b, 2011, 2014; Wong et al., 2017; Wood, 2012).

Glycerophospholipids (GP), as the most abundant structural components within cellular membranes, regulate membrane mobility, provide secondary messengers for cellular signalling, play a critical role in synaptic transmission between neurons and can cause neuronal death when dysregulated (Bennett et al., 2013; Wong et al., 2017). The homeostasis of GP has been reported to be altered in $\mathrm{AD}$, with a general downregulation of glycerophosphocholine $(\mathrm{PC})$, glycerophosphoethanolamine (PE) and glycerophosphoinositol (PI) species (Bennett et al., 2013; Kosicek \& Hecimovic, 2013; Wong et al., 2017; Wood, 2012), with additional alterations in a specific GP subclass, namely alkenyl (i.e., plasmalogen)-containing species in AD brain (Ginsberg et al., 1995; Han et al., 2001; Pettegrew et al., 2001).

Sphingolipids (SP), are another fundamental structural component of cellular membranes, and composed of sphingomyelin (SM), ceramide (Cer) and other glycosphingolipid species, play a critical role in the central nervous system (CNS) and are heavily involved in neuronal signalling (Wood, 2012). SM and Cer are major components of the myelin sheath with deregulation of the $\mathrm{SM} /$ Cer signalling cascade suggested to cause synaptic dysfunction, neuro-inflammation and neuronal apoptosis in AD (Cutler et al., 2004; Haughey et al., 2010; He et al., 2010; Mielke \& Lyketsos, 2010; Satoi et al., 2005; Wong et al., 2017; Wood, 2012).

Exosomes are small extracellular vesicles (EVs) released from cells upon fusion of the multi-vesicular body (MVBs) with the plasma membrane (Johnstone et al., 1987; Raposo et al., 1996). They are surrounded by a lipid bilayer, and packaged with cargoes including proteins, lipids, nucleic acids and metabolites which reflect their cellular origin and mirror the physiological or pathological condition of the parental cell (Kanninen et al., 2016). EVs have a unique lipid composition with multiple studies reporting enrichment of specific lipid classes including SM, glycerophosphoserine (PS), and PC and PE ether lipids, in EVs relative to their parental origin (Hessvik \& Llorente, 2017; Kanninen et al., 2016; Laulagnier et al., 2004; Llorente et al., 2013; Lydic et al., 2015; Phuyal et al., 2015; Record et al., 2014, 2018; Simbari et al., 2016). EVs are regarded as critical players in intercellular communication by inducing phenotypic changes and altering homeostasis in recipient cells upon fusion or uptake (Rai et al., 2019; Record et al., 2014; Simons \& Raposo, 2009). Given EVs are present in the extracellular environment, including body fluids, there is intense interest in using EVs as a source of tissue and disease specific biomarkers. Recent studies have revealed exosomal proteins as potential biomarkers in non-small cell lung cancer and pancreatic cancer (Li et al., 2017; Melo et al., 2015) and certain lipid species in urinary exosomes have been reported to serve as biomarkers for prostate cancer diagnosis (Skotland et al., 2017a). While the proteomic and nucleic acid compositions of EVs have been extensively studied, lipidomic profiling of EVs is still in its infancy (Skotland et al., 2020) even though lipids are an essential component of EVs.

As BDEVs are predicted to participate in AD pathogenesis (DeLeo \& Ikezu, 2018; Kanninen et al., 2016; Rajendran et al., 2006; Sharples et al., 2008; Thompson et al., 2016; Vella et al., 2016; Vingtdeux et al., 2012), we hypothesized that EVs could provide a rich source of lipids that reflect alterations observed in the CNS in AD (He et al., 2010; Wood, 2012). Here, using methods we previously developed for the isolation and enrichment of EVs from human brain tissue (Vella et al., 2017), coupled with semiquantitative ultrahigh resolution and accurate mass spectrometry (UHRAMS) based lipidome analysis (Ryan \& Reid, 2016), we have determined, for the first time, the lipid composition of BDEVs in human frontal cortex and identified the lipidomic signature of EVs in human AD compared to gender and age-matched controls (CTL).

\section{2 | MATERIALS AND METHODS}

\subsection{Human frontal cortices}

Fresh frozen human post-mortem frontal cortex tissues of $\mathrm{n}=8 \mathrm{AD}$ male subjects (mean age $74.5 \pm$ SD 7.0 years) and $\mathrm{n}=8$ gender and age-matched CTL subjects (mean age $73.5 \pm$ SD 5.9 years) with no evidence of dementia, stored at $-80^{\circ} \mathrm{C}$, were obtained from the Victoria Brain Bank. The average post-mortem delay before tissue collection was $23.3 \pm 17.4 \mathrm{~h}$ for $\mathrm{AD}$ and $42 \pm 16.3 \mathrm{~h}$ for CTL. Individual tissue case information, including age, PMI and ApoE genotype, is provided in Supplemental Table 1. Tissue handling and experimental procedures were approved by The University of Melbourne human ethics committee and in accordance with the National Health and Medical Research Council guidelines. 


\section{2 | BDEV enrichment}

The BDEV isolation method was modified from our previously published protocol (Vella et al., 2017). A schematic of the workflow is shown in Supplemental Figure 1. Frozen frontal cortex tissues (approximately $2 \mathrm{~g}$ ) were sliced lengthways on ice to generate 1-2 cm long, 2-3 mm wide tissue sections. Approximately $30 \mathrm{mg}$ tissue pieces from each sample ("Brain Total") were collected, weighed and placed in 19x volume of tissue weight of Dulbecco's phosphate buffered saline (DPBS, Thermo Fisher Scientific) solution containing 1x PhosSTOP phosphatase inhibitor (Sigma Aldrich) / cOmplete protease inhibitor (including EDTA, Sigma Aldrich) for immunoblot analysis. The remaining cut tissue sections were weighed and incubated with $50 \mathrm{U} / \mathrm{ml}$ collagenase type 3 (\#CLS-3, CAT\#LS004182, Worthington) digestion buffer (at ratio of $8 \mu \mathrm{l} / \mathrm{mg}$ tissue) in a shaking water bath $\left(25^{\circ} \mathrm{C}\right.$, a total of $20 \mathrm{~min}$ ). During incubation, tissue slices were inverted twice at the 10-min time point, gently pipetted up and down twice at the 15-min time point and then allowed incubation for a further $5 \mathrm{~min}$, followed by the addition of ice-cold 10x inhibition buffer, which was made of 10x phosphatase inhibitor and 10x protease inhibitor in DPBS. The final concentration of inhibition buffer in solution was $1 \mathrm{x}$.

The dissociated tissue in solution was subjected to a series of centrifugations, including a $300 \times g, 4^{\circ} \mathrm{C}$ for $5 \mathrm{~min}$, a $2000 \times g$, $4^{\circ} \mathrm{C}$ for $10 \mathrm{~min}$ and a $10,000 \times g, 4^{\circ} \mathrm{C}$ for $30 \mathrm{~min}$. Representative $300 \times g$ pellets were collected ('Brain $+\mathrm{C}^{\prime}$ for collagenase treatment) and either placed in $19 \times$ volume of tissue weight of DPBS with $1 \times$ phosphatase inhibitor / protease inhibitor solution for protein quantification and immunoblot analysis or combined with 19x volume of tissue weight of ice-cold $60 \%$ methanol (LCMS grade, EMD Millipore Corporation) containing 0.01\% (w/v) butylated hydroxytoluene (BHT, Sigma Aldrich) for lipid extraction. The $10,000 \times g$ supernatant was loaded on top of the triple sucrose density gradient $(0.6 \mathrm{M}, 1.3 \mathrm{M}, 2.5 \mathrm{M})$ as indicated in the method (Vella et al., 2017) in ultra-clear SW40Ti tubes (Beckman Coulter). The sucrose gradients were centrifuged at $200,000 \mathrm{x}$ g avg at $4^{\circ} \mathrm{C}$ for 173 min using a SW40Ti rotor (Beckman Coulter). After the spin, the three fractions (F1, F2 and F3, $1.2 \mathrm{ml}$ each) were sequentially collected and refractive index was measured. Each fraction was subjected to a wash spin in ice-cold DPBS at $128,000 \times g$ avg, at $4^{\circ} \mathrm{C}$ for 80 min using a F37L- $8 \times 100$ rotor (Thermo Fisher Scientific). The pelleted EVs were resuspended in $150 \mu \mathrm{l}$ ice-cold DPBS with 1x phosphatase inhibitor / protease inhibitor solution. In each $150 \mu \mathrm{l}$ vesicle suspension, $5 \mu \mathrm{l}$ were aliquoted for TEM, $65 \mu \mathrm{l}$ were aliquoted for protein quantification and immunoblot analysis and $80 \mu \mathrm{l}$ were used for lipid extraction.

\subsection{Homogenization and protein quantification for immunoblot}

Tissue in DPBS solution with 1x phosphatase inhibitor / protease inhibitor was homogenised for $12 \mathrm{~s}$ at $50 \%$ intensity using a tapered microtip attached to the Sonifier Cell Disruptor (Branson) and sonicated in ice-cold water bath for 20 min before being submitted to clarification spin $\left(10,000 \times g, 10 \mathrm{~min}, 4^{\circ} \mathrm{C}\right)$. The EV suspensions were sonicated in an ice-cold water bath sonicator for $20 \mathrm{~min}$. Protein content in tissue homogenates and EV suspensions was determined by Pierce ${ }^{\mathrm{TM}}$ BCA assay kit (Thermo Fisher Scientific) according to the manufacturer's instructions.

\section{$2.4 \quad$ SDS-PAGE and Western Blot (WB) analysis}

Samples were prepared in $4 \times$ Laemmli sample buffer, then boiled $\left(10 \mathrm{~min}, 90^{\circ} \mathrm{C}\right)$ followed by centrifugation $(14,000 \times g, 1 \mathrm{~min})$. Normalised samples $(1-3 \mu \mathrm{g}$ ) were electrophoresed on 12\% Mini-Protean TGX Stain-Free gels (BioRad) or 4\%-20\% Criterion TGX Stain-Free Precast gels (BioRad) in Tris/Glycine/SDS running buffer (BioRad) for $30 \mathrm{~min}$ at $245 \mathrm{~V}$. Proteins were transferred onto nitrocellulose membranes using either a Trans-Blot Turbo Transfer System (7 min, 25V, BioRad) or an iBlot 2 Dry Blotting System (P0 method, Thermo Fisher Scientific). Membranes were blocked with 5\% (w/v) skim milk in TBS-T ( $1 \mathrm{~h}$, room temperature) followed by overnight incubation with primary antibodies (calnexin \#ab22595 from Abcam, syntenin \#ab133267 from Abcam, TSG101 \#T5701 from Sigma) at $4^{\circ} \mathrm{C}$. Membranes were washed 4 times with TBS-T (30 min, room temperature) following a $1 \mathrm{~h}$, room temperature incubation of the secondary anti-rabbit antibody either conjugated to HRP (\#7074S from Cell Signalling Technology) or the IRDye 800CW Goat anti-Rabbit IgG secondary antibody (\#925-32211, LICOR). All antibodies were diluted in 5\% (w/v) skim milk in TBS-T. Membranes were washed 4 times with TBS-T (30 min, room temperature). The membranes were visualised either on a ChemiDoc MP Imager (BioRad) following development with Clarity Western Enhanced Chemiluminescence Blotting Substrate (BioRad) or on an Odyssey Fc Imaging System (LI-COR).

\section{$2.5 \quad$ Transmission electron microscopy (TEM)}

A $5 \mu \mathrm{l} \mathrm{EV}$ suspension from F1, F2 or F3 in 1\% (w/v) electron microscopy-grade glutaraldehyde was absorbed onto neutralised 300mesh carbon-coated formvar copper grids (ProSciTech, QLD, Australia) for $5 \mathrm{~min}$. Excessive liquid was removed and grids were 
washed with DPBS and MilliQ water, and negatively stained with 2\% (w/v) saturated aqueous uranyl acetate for 12 sec. Excessive stain was removed and grids were dried. Images were taken on a Tecnai G2 F30 (FEI, Eindhoven, The Netherlands) transmission electron microscope operating at $300 \mathrm{kV}$. Wide field images encompassing multiple vesicles were captured to provide an overview of the fraction in addition to close-up images. Electron microscopy was performed in the Bio21 Advanced Microscopy Facility, Bio21 Molecular Science and Biotechnology Institute, at The University of Melbourne.

\subsection{Monophasic lipid extraction from tissues and derived extracellular vesicles}

The "total brain with collagenase" tissue pellets in ice-cold $60 \%$ methanol containing $0.01 \%(\mathrm{w} / \mathrm{v})$ BHT were homogenised using a cell disrupter as described above. $100 \mu \mathrm{l}$ of the homogenates were combined with $100 \mu \mathrm{l}$ of $60 \%$ methanol containing $0.01 \%$ (w/v) BHT. $80 \mu \mathrm{l}$ of the F2 BDEV suspensions were combined with $20 \mu \mathrm{l}$ of ice-cold methanol with $0.1 \%$ (w/v) BHT and $100 \mu$ l of ice-cold methanol to make a final volume of $200 \mu \mathrm{l} 60 \%$ methanol containing $0.01 \%(\mathrm{w} / \mathrm{v}) \mathrm{BHT}$. All samples were sonicated in an ice-cold water bath sonicator $(20 \mathrm{~min}$ ) prior to lipid extraction. Monophasic lipid extraction followed the method previously reported by Lydic et al. (Lydic et al., 2015) with modification as described below. $120 \mu \mathrm{l}$ of MilliQ water, $420 \mu \mathrm{l}$ of methanol with $0.01 \%$ (w/v) $\mathrm{BHT}$, and $270 \mu \mathrm{l}$ of chloroform were added to all samples. For every $10 \mu \mathrm{g}$ protein present in the samples, $1 \mu \mathrm{l}$ of a customised isotope labelled internal standard lipid mixture and $1 \mu \mathrm{l}$ of a d5-TG Internal Standard Mixture I (Avanti Polar Lipids, Alabaster, AL, USA) were added. The customised isotope labelled internal standard mixture was comprised of 14 deuterated lipid standards (Avanti Polar Lipids, Alabaster, AL, USA): 15:0-18:1(d7) PC (250 $\mu \mathrm{M}), 15: 0-18: 1(\mathrm{~d} 7)$ PE $(240 \mu \mathrm{M}), 15: 0-18: 1(\mathrm{~d} 7)$ PS $(250 \mu \mathrm{M})$, 15:0-18:1(d7) PG $(20 \mu \mathrm{M}), 15: 0-18: 1(\mathrm{~d} 7)$ PI $(220 \mu \mathrm{M}), 15: 0-18: 1(\mathrm{~d} 7)$ PA $(180 \mu \mathrm{M}), 18: 1(\mathrm{~d} 7)$ LPC $(45 \mu \mathrm{M}), 18: 1(\mathrm{~d} 7)$ LPE $(10 \mu \mathrm{M})$, 18:1(d7) Chol Ester $(10 \mu \mathrm{M}), 18: 1(\mathrm{~d} 7)$ MG $(10 \mu \mathrm{M}), 15: 0-18: 1(\mathrm{~d} 7)$ DG $(17 \mu \mathrm{M}), 18: 1(\mathrm{~d} 9)$ SM $(80 \mu \mathrm{M})$, d18:1(d7)-15:0 Cer $(40 \mu \mathrm{M})$ and Cholesterol(d7) $(20 \mu \mathrm{M})$. The d5-TG Internal Standard Mixture I contained 20:5-22:6-20:5 (d5) TG (4.03 $\mu \mathrm{M}), 14: 0-16: 1-14: 0$ (d5) TG (3.99 $\mu \mathrm{M}), 15: 0-18: 1-15: 0$ (d5) TG (3.97 $\mu \mathrm{M}), 16: 0-18: 0-16: 0$ (d5) TG (4.05 $\mu \mathrm{M})$, 17:0-17:1-17:0 (d5) TG (4.14 $\mu \mathrm{M}), 19: 0-12: 0-$ 19:0 (d5) TG (4.01 $\mu \mathrm{M}), 20: 0-20: 1-20: 0$ (d5) TG (3.81 $\mu \mathrm{M})$, 20:2-18:3-20:2 (d5) TG (3.96 $\mu \mathrm{M})$, 20:4-18:2-20:4 (d5) TG (3.90 $\mu \mathrm{M})$. The 15:0-18:1-15:0 (d5) TG was used for semi-quantification of endogenous TG lipids. Samples were vortexed thoroughly and incubated with 1,000 rpm shaking at room temperature for $30 \mathrm{~min}$, followed by centrifugation at $14,000 \mathrm{rpm}$ at room temperature for $15 \mathrm{~min}$. Supernatants containing lipids were transferred to new tubes. The remaining pellets were re-extracted with $100 \mu \mathrm{L}$ of MilliQ water and $400 \mu \mathrm{l}$ of chloroform:methanol (1:2, v:v) containing $0.01 \%$ (w/v) butylated hydroxytoluene (BHT) following incubation and centrifugation as described above. The supernatants from the repetitive extractions were collected and pooled, dried by evaporation under vacuum using a GeneVac miVac sample concentrator (SP Scientific, Warminster, PA, USA) and then reconstituted in isopropanol:methanol:chloroform (4:2:1, v:v:v, containing 0.01\% BHT) at a final concentration of $4 \mu \mathrm{l}$ lipid extract per $\mu$ g protein.

\section{7 | Sequential functional group derivatization of aminophospholipids and plasmalogen-containing lipids}

Derivatization of aminophospholipids (i.e., PE and PS) and plasmalogen-containing lipids followed the method previously reported by Ryan and Reid (2016). Prior to derivatization, a $2.5 \mathrm{mM}$ stock solution of triethyamine (TEA) in chloroform was freshly prepared by adding $3.4 \mu \mathrm{l}$ TEA to $10 \mathrm{ml}$ of chloroform. A $2.5 \mathrm{mM}$ stock solution of S,S'dimethylthiobutanoylhydroxysuccinimide ester iodide $\left({ }^{13} \mathrm{C}_{1}\right.$-DMBNHS) was freshly prepared by dissolving $4.87 \mathrm{mg}{ }^{13} \mathrm{C}_{1^{-}}$ DMBNHS in $5 \mathrm{ml}$ of dimethylformamide (DMF). A stock solution of $3.94 \mathrm{mM}$ iodine was freshly prepared by dissolving $10 \mathrm{mg}$ iodine in $10 \mathrm{ml}$ chloroform. A stock solution of $90 \mathrm{mM}$ ammonium bicarbonate was freshly prepared by dissolving $35.6 \mathrm{mg}$ ammonium bicarbonate in $5 \mathrm{ml}$ of HPLC methanol. A solution of 2:1 (v:v) chloroform:methanol containing $266 \mu \mathrm{M}$ iodine and $2 \mathrm{mM}$ ammonium bicarbonate was prepared by adding $160 \mu \mathrm{l}$ of $3.94 \mathrm{mM}$ iodine in chloroform to $1.44 \mathrm{ml}$ chloroform, and $53.3 \mu \mathrm{l}$ of $90 \mathrm{mM}$ ammonium bicarbonate in methanol to $746.7 \mathrm{ml}$ methanol, then combined and placed in an ice bath. Due to the limitations in sample amounts, no replicate derivatization reactions were performed. $4 \mu$ l of brain tissue or BDEV lipid extracts were aliquoted to individual wells of a Whatman Multi-Chem 96-well plate (Sigma Aldrich, St. Louis, MO, USA). The solvent was evaporated under vacuum with a GeneVac miVac sample concentrator. $40 \mu \mathrm{l}$ of a solution of 39:1.1:1 (v:v:v) chloroform:2.5 mM TEA:2.5 mM ${ }^{13} \mathrm{C}_{1}$-DMBNHS reagent was added to each dried lipid extract and the 96 -well plate was sealed with Teflon Ultra Thin Sealing Tape.

Samples were then incubated at room temperature with gentle shaking for $30 \mathrm{~min}$. After incubation, the solvents were evaporated under vacuum with a GeneVac miVac sample concentrator and samples were chilled on ice for 10 min prior to addition of $40 \mu \mathrm{l}$ of the 2:1 (v:v) chloroform:methanol containing $266 \mu \mathrm{M}$ iodine and $2 \mathrm{mM}$ ammonium bicarbonate. Reactions were mixed by careful pipetting and the plate was sealed with aluminium foil and then placed on ice for 5 min before solvents were completely removed by evaporation under vacuum with a GeneVac miVac sample concentrator. The dried lipid extracts were washed three times with $40 \mu \mathrm{l}$ of $10 \mathrm{mM}$ aqueous ammonium. Remaining traces of water were then removed by evaporation under vacuum 
with a GeneVac miVac sample concentrator. The derivatized brain tissue lipid extracts and BDEV lipid extracts were then resuspended in $50 \mu \mathrm{l}$ and $25 \mu \mathrm{l}$ of isopropanol:methanol:chloroform (4:2:1, v:v:v) containing $20 \mathrm{mM}$ ammonium formate respectively. The 96-well plate was then sealed with Teflon Ultra Thin Sealing Tape prior to mass spectrometry analysis.

\section{8 | Direct infusion nano-electrospray ionization (nESI) - ultrahigh resolution and accurate mass spectrometry (UHRAMS) and higher energy collision induced dissociation tandem mass spectrometry (HCD-MS/MS) shotgun lipidome analysis}

For underivatized samples, $4 \mu \mathrm{l}$ of brain tissue or BDEV lipid extracts were aliquoted in triplicate to individual wells of a twin-tec 96-well plate (Eppendorf, Hamburg, Germany). The brain tissue lipid extracts and BDEV lipid extracts were dried and then resuspended in $50 \mu \mathrm{l}$ (brain tissue) and $25 \mu \mathrm{l}$ (BDEV) of isopropanol:methanol:chloroform (4:2:1, v:v:v) containing $20 \mathrm{mM}$ ammonium formate respectively. The 96-well plate was then sealed with Teflon Ultra Thin Sealing Tape prior to mass spectrometry analysis. $10 \mu \mathrm{l}$ of each underivatized or derivatized lipid sample was aspirated and introduced via nano-ESI to an Orbitrap Fusion Lumos mass spectrometer (Thermo Fisher Scientific, San Jose, CA, USA) using an Advion Triversa Nanomate (Advion, Ithaca, NY, USA) operating with a spray voltage of $1.1 \mathrm{kV}$ and a gas pressure of $0.3 \mathrm{psi}$ in both positive and negative ionization modes. For MS analysis, the RF lens was set at $10 \%$. Full scan mass spectra were acquired at a mass resolving power of 500,000 (at $200 \mathrm{~m} / \mathrm{z})$ across a $\mathrm{m} / \mathrm{z}$ range of $350-1600$ using quadrupole isolation, with an automatic gain control (AGC) target of 5e5. The maximum injection time was set at $50 \mathrm{~ms}$. Spectra were acquired and averaged for $3 \mathrm{~min}$. Following initial 'sum-composition' lipid assignments by database analysis (see below), 'targeted' higher-energy collision induced dissociation (HCD-MS/MS) product ion spectra were acquired on selected precursor ions at a mass resolving power of 120,000 and default activation times in positive ionization mode using the underivatized lipid extracts to confirm the identities of lipid head groups, or in negative ionization mode using underivatized lipid extracts for fatty acid chain identification. HCD-MS/MS collision energies were individually optimized for each lipid class of interest using commercially available lipid standards whenever possible.

\subsection{Lipid identification and quantification and data analysis}

'Sum composition' level lipid identifications were achieved using a developmental version of LipidSearch software 5.0 $\propto$ (Mitsui Knowledge Industry, Tokyo, Japan) by automated peak peaking and searching against a user-defined custom database of lipid species (including the deuterated internal standard lipid species and allowing for the mass shifts introduced by ${ }^{13} \mathrm{C}_{1}$-DMBNHS and iodine/methanol derivatization). The parent tolerance was set at $3.0 \mathrm{ppm}$, a parent ion intensity threshold three times that of the experimentally observed instrument noise intensity, and a max isotope number of 1 (i.e., matching based on the monoisotopic ion and the $\mathrm{M}+1$ isotope), a correlation threshold (\%) of 0.3 and an isotope threshold (\%) of 0.1. The lipid nomenclature used here follows that defined by the LIPID MAPS consortium (Fahy et al., 2005). Semi-quantification of the abundances of identified lipid species was performed using an in-house R script, by comparing the identified lipid ion peak areas to the peak areas of the internal standard for each lipid class or subclass, followed by normalization against the total protein amount in the samples.

\subsection{Data analysis}

For lipid identification obtained from derivatized samples, the mean normalized abundances of each lipid species were included only if the lipid was identified in at least 6 out of 8 biological samples. For underivatized samples, the mean normalized abundances were included only if the lipid was identified in all three measurement replicates and detected in at least 6 out of 8 biological samples.

The number of identified lipid species in frontal cortex at the lipid category, class or subclass levels, and their mean summed lipid abundances were normalized to tissue weight (i.e., $\mathrm{pmol} / \mathrm{mg}$ tissue). Comparisons of frontal cortex to their respective BDEVs were normalized to either the total lipid concentration (i.e., mol\% total lipid), or total lipid-class concentration (i.e., mol\% total lipid class) to reduce the dependence on starting tissue weight given the inter-experimental variability of enzymatic tissue digestion on BDEV yield. Absolute lipid abundance normalized to protein (i.e., $\mathrm{pmol} / \mu \mathrm{g}$ protein) or total lipid concentration (i.e., mol\% total lipid) or total lipid class concentration (i.e., mol\% class) was used when comparing AD and CTL BDEVs.

Significant differences in the mean normalized abundances, at the lipid category, class, subclass or individual 'sumcomposition' lipid species levels, in CTL tissue vs. AD tissue, CTL tissue vs. CTL BDEV, AD tissue vs. AD BDEV and CTL BDEV vs. AD BDEV, were determined by ANOVA followed by Sidak's multiple comparisons test using the GraphPad Prism 8.0 software, with multiplicity adjusted $\mathrm{p}$ value $<0.01$. 
A

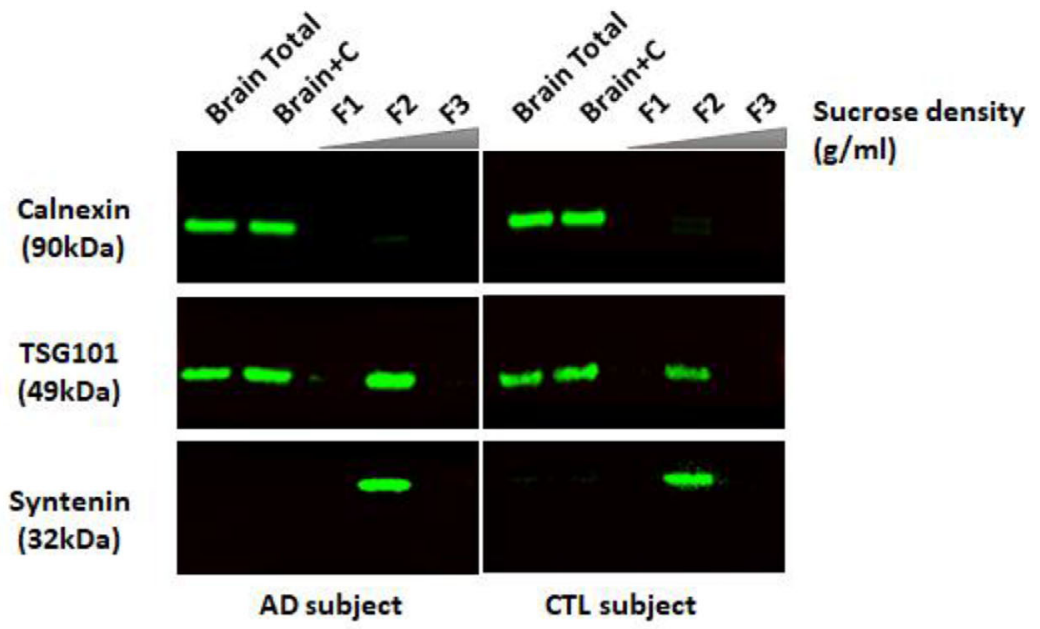

B

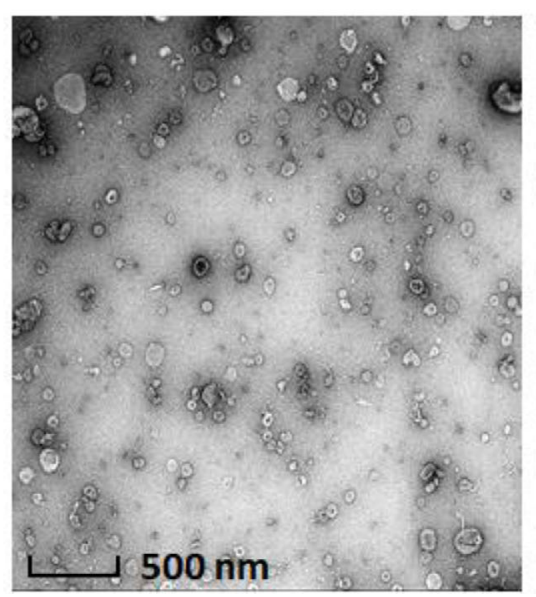

F I G U R E 1 Characterization of BDEVs from human frontal cortex. [(A) Western blot analysis. Equivalent amount of protein from human frontal cortex brain homogenates (Brain Total), brain homogenates after collagenase treatment $($ Brain $+\mathrm{C})$ and BDEV suspensions (F1, F2, and F3) were subjected to SDS-PAGE. Total proteins were visualized using stain-free technology to ensure similar loading. Frontal cortex brain tissue homogenates, 'Brain Total' and 'Brain $+\mathrm{C}$, were enriched in calnexin, while calnexin was not detectable in an equivalent amount of BDEV protein. Proteins typical of endosome derived exosomes, TSG101 and syntenin, were observed in $\mathrm{F} 2$, illustrating that $\mathrm{F} 2$ is enriched in exosome-like vesicles. The densities in F1, F2 and F3 were approximately $1.02 \mathrm{~g} / \mathrm{ml}, 1.08 \mathrm{~g} / \mathrm{ml}$ and $1.17 \mathrm{~g} / \mathrm{ml}$ respectively. Immunoblots images are representative of 8 independent CTL and $8 \mathrm{AD}$ human tissue samples. (B) Transmission electron microscopy (TEM) of BDEV. All BDEVs from F2 were fixed with $1 \%(\mathrm{w} / \mathrm{v})$ glutaraldehyde, negatively stained with $2 \%(\mathrm{w} / \mathrm{v})$ uranyl acetate and visualized by a FEI Tecnai F30 transmission electron microscope. The zoomed-out image (left) provides an overview of the BDEV suspensions with scale bar representing $500 \mathrm{~nm}$. The close-up image (right) shows clearer small, cup-shaped BDEVs which is consistent with the morphology of vesicles with scale bar representing $200 \mathrm{~nm}$. The TEM images of BDEVs in $\mathrm{F} 2$ are representative of the BDEVs from all samples (AD and CTL). $\mathrm{AD}=$ Alzheimer's disease, $\mathrm{CTL}=$ control, $\mathrm{BDEV}=$ brain derived extracellular vesicles]

\section{3 | RESULTS}

\subsection{Characterization of BDEVs}

We recently reported a method to isolate small extracellular vesicles from post-mortem human frontal cortex, that contain the hallmarks of endosomal derived exosomes (Cheng et al., 2020; Vella et al., 2017). These vesicles fulfilled the experimental requirements as set out by the International Society for Extracellular Vesicles 2018 guidelines (Théry et al., 2018). Here, we employed this method to isolate BDEVs from post-mortem frontal cortex brain tissue obtained from AD and CTL subjects. Dissociated collagenase treated tissue ("Brain $+C$ ") was subject to sequential centrifugation and the 10,000 $\mathrm{x}$ g supernatant was loaded on top of a triple sucrose density fraction to separate EVs based on density (Supplemental Figure 1). Factors such as post-mortem delay and storage can negatively impact tissue quality, resulting in contamination of EV pellets with cellular debris (Vella et al., 2017). We previously reported that immunoblotting provides the most robust quality-control measure for tissue EV isolation, with proteins such as calnexin providing a useful indicator of EV purity (Vella et al., 2017). Consequently, all BDEVs isolated as part of the current study were subject to western blotting and TEM (representative images in Figures 1A and 1B) to screen for EV markers and contaminants before subjecting samples to downstream lipidomic analysis. As per our previous studies (Vella et al., 2017), small EVs were identified in fraction 2 (F2) with a density of approximately $1.08 \mathrm{~g} / \mathrm{cm}^{3}$. EVs in F2 were enriched in the small EV-specific markers TSG101 and syntenin, and depleted of calnexin (Figure 1A). TEM images of F2 showed small, cup-shaped membrane vesicles with a diameter of 40-200 nm for both AD and CTL samples (Figure 1B). Together, these results suggest that F2 contains vesicles that are consistent with the density, morphology, size and protein co-enrichment of endosome derived small BDEVs as we have previously reported. Supplemental Table 2 shows the protein content determined for each tissue and BDEV fraction, where on average, AD subjects yielded approximately half the BDEV yield compared to CTL subjects, a possible reflection of the cellular dysfunction occurring in late stage disease. 

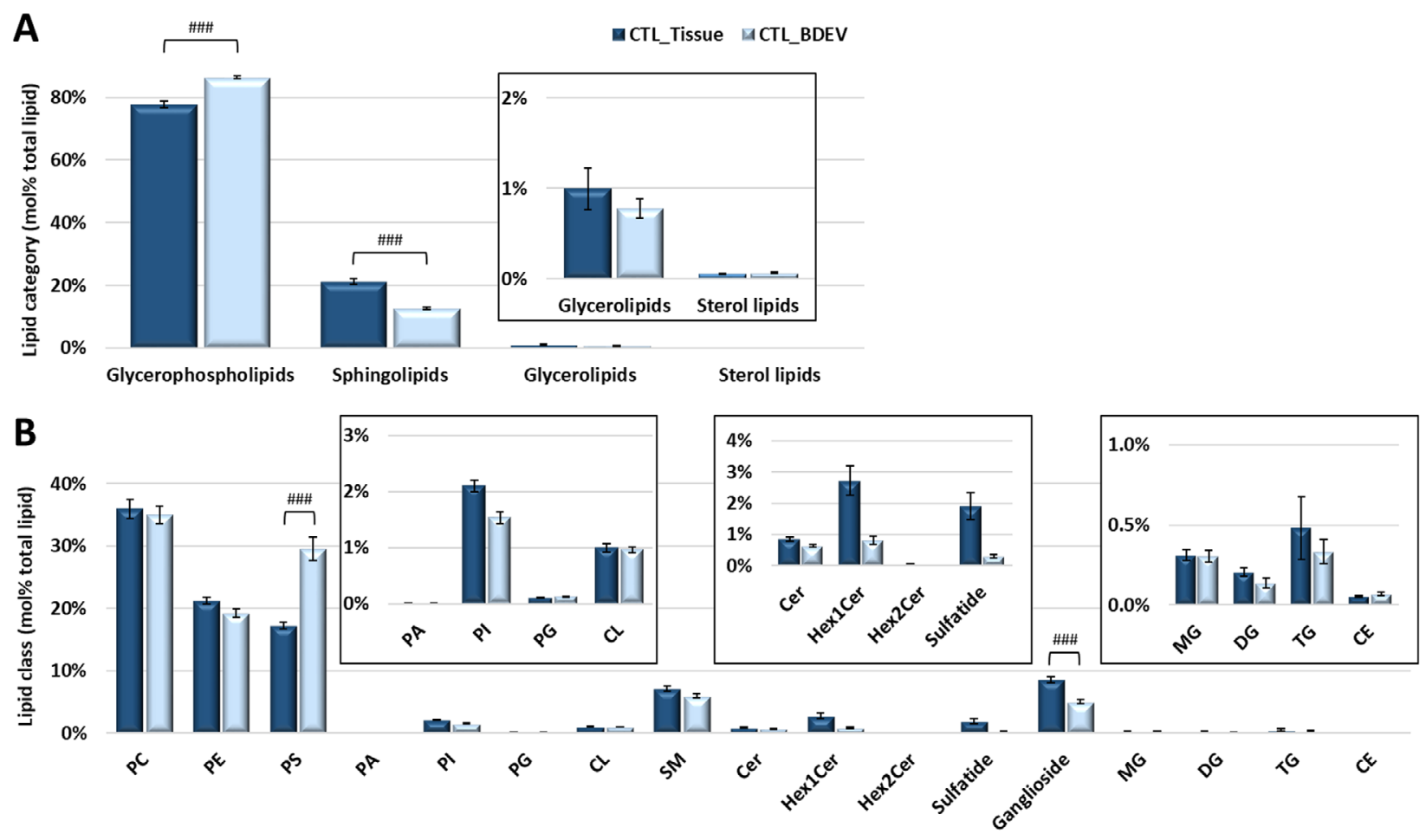

F I G U RE 2 Comparison of total lipid abundance between frontal cortex and the BDEVs in this tissue from control subjects. [(A) Mol\% total lipid abundance distributions at the lipid category level. Four lipid categories, covering glycerophospholipids (GPs), sphingolipids (SPs), glycerolipids (GLs) and sterol lipids (STs) were included in this study. The inset shows the low abundant GL and ST categories for clarity. BDEVs contained significantly higher levels of GPs and corresponding lower levels of SPs in comparison to tissue. (B) Mol\% total lipid abundance distributions at the lipid class level. A total of 17 lipid classes were identified in this study. The inset shows the low abundant PA, PI, PG, CL, Cer, Hex1Cer, Hex2Cer, sulfatide, MG, DG, TG and CE classes for clarity. BDEVs were found to be significantly enriched in PS lipids, making up approx. 30\% of the total lipid abundance, compared to tissue (approx. 17\%). Ganglioside lipids were significantly downregulated in BDEV compared to tissue. Data represent the average mol\% total lipid abundances \pm standard error of the mean. Statistical significance was determined by ANOVA followed by Sidak's multiple comparison test, with multiplicity adjusted p value $<0.01$. \#\#\# Adjusted $P$ value $<0.0001$. CTL $=$ control, $\mathrm{BDEV}=$ brain derived extracellular vesicles. $\mathrm{N}=8$ CTL subjects $]$

\subsection{Lipidome analysis of frontal cortex and derived BDEVs}

To investigate the lipid composition of frontal cortex tissue and the BDEVs isolated from this tissue, an in-depth semi-quantitative mass spectrometric analysis was performed. In total, 692 lipid molecules from four main lipid categories, including glycerophospholipids (GP), sphingolipids (SP), glycerolipids (GL) and sterol lipids (ST), covering 17 lipid classes, were identified and semiquantified at the 'sum composition' level (i.e., where the identity of the lipid class, subclass and the total number of carbon atoms and total number of $\mathrm{C}=\mathrm{C}$ double bonds in the fatty acyl/alkyl/alkenyl moieties are assigned. For example, PE(P-38:4) indicates a 1-(1Z-alkenyl), 2-acylglycerophosphoethanolamine lipid containing a total of 38 carbons and four double bonds in the alkenyl and acyl chains). Initial lipidome analysis comparing "Brain Total" and "Brain+C" indicated that collagenase did not result in any significant alterations in the number or abundance of the identified lipids in brain tissue (data not shown). Therefore, all subsequent comparisons were performed using "Brain+C" samples. The number of identified lipid species at the lipid category, class or subclass levels, and their mean summed lipid abundances, either normalized to tissue weight (i.e., $\mathrm{pmol} / \mathrm{mg}$ tissue), protein content (i.e., pmol $/ \mu$ g protein), total identified lipid concentration (i.e., mol\% total lipid), total identified lipidcategory concentration (i.e., mol\% category), or total identified lipid class concentration (i.e., mol\% class) are summarised in Supplemental Table 3. A complete list of the individual identified lipids and their abundances in tissue and BDEVs can be found in Supplemental Table 4.

No difference was observed at the total lipid level, lipid category or lipid class levels of annotation between disease and CTL frontal cortex (i.e., pmol/mg tissue) (Supplemental Table 3 and Supplemental Figure 2). We then determined the lipid profile of BDEVs isolated from this frontal cortex tissue and compared to the tissue profile. Figure 2A and Supplemental Figure 3A show the mean of the summed lipid abundances at the lipid category in CTL and AD subjects respectively. Features common to BDEVs, independent of diagnosis, included significantly higher GP $(P<0.0001$, mol\%) and lower SP $(P<0.0001$, mol\%) compared to parental tissue. At the lipid class level, BDEVs were significantly enriched in PS $(P<0.0001, \mathrm{~mol} \%)$ with a corresponding decrease in ganglioside lipids $(P<0.0001, \mathrm{~mol} \%)$ (Figure 2B and Supplemental Figure 3B). Encouragingly, the BDEVs were not abundant 

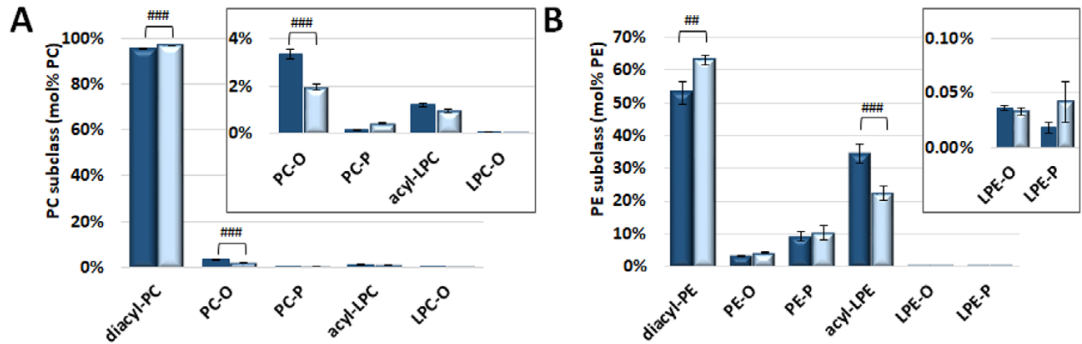

a CTL_Tissue

$\triangle$ CTL_BDEV
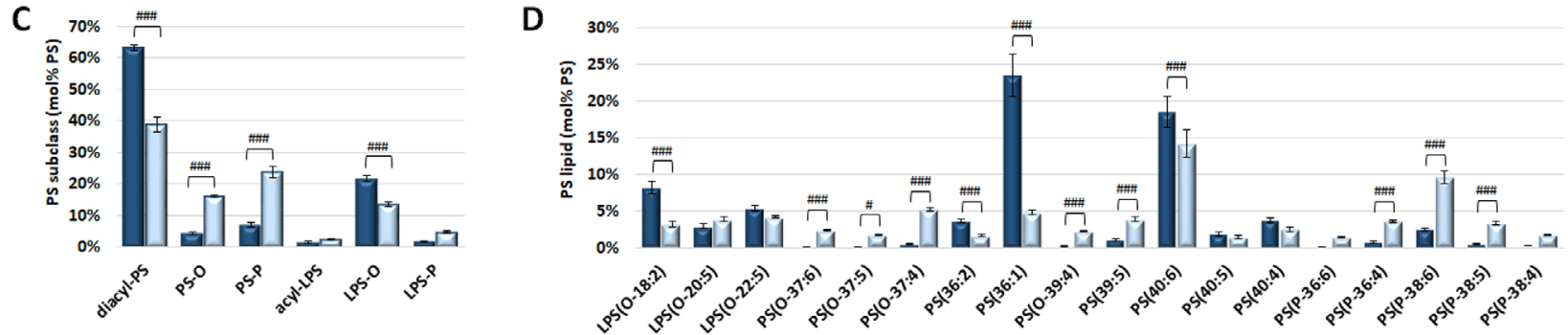

F I G U R E 3 Comparison of PC, PE and PS lipid subclasses abundance and individual PS lipid molecules between tissue and BDEVs. [(A) Mol\% total PC lipid subclass abundance distributions. A significant increase in diacyl-PC was observed in BDEV vs. tissue, accompanied with a decrease in PC-O. The inset shows the low abundant PC-O, PC-P, acyl-LPC and LPC-O for clarity. (B) Mol\% total PE lipid subclass abundance distributions. Significant increase in diacyl-PE was observed in BDEV, accompanied with decreased in acyl-LPE. (C) Mol\% total PS lipid subclass abundance distributions. A significant decrease was observed in diacyl-PS and LPS-O in BDEV relative to tissue, accompanied with an enrichment of PS-O and PS-P. (D) Mol\% total PS lipid abundance distributions of individual PS molecules. Diacyl PS species, PS(36:2), PS(36:1) and PS(40:6) were significantly decreased in BDEV relative to tissue. An overall increase in ether PS species, including PS(O-37:6), PS(O-37:5), PS(O-37:4), PS(O-39:4), PS(P-36:4), PS(P-38:6) and PS(P-38:5), was observed in BDEV. Only the most abundant lipid molecules in PS lipid class are shown for clarity. Data represent the average mol\% total lipid class abundance \pm standard error of the mean. Statistical significance was determined by ANOVA followed by Sidak's multiple comparison test, with multiplicity adjusted $P$ value $<0.01$. ${ }^{*}$ Adjusted $P$ value $<0.01, \#$ adjusted $P$ value $<0.001$, and ${ }^{\# \#}$ adjusted $P$ value $<0.0001$. CTL $=$ control, BDEV $=$ brain derived extracellular vesicles. $\mathrm{N}=8 \mathrm{CTL}$ subjects $]$

in GL or cholesterol ester (CE) lipids (Figure 2 and Supplemental Figure 3), indicating limited lipoprotein contamination (Serna et al., 2015; Sun et al., 2019; Wang \& Eckel, 2014). Cardiolipins (CL), which reside on the inner membrane of mitochondria, were also limited in abundance (Figure 2B and Supplemental Figure 3B), further validating our methodological approach for isolating EVs from tissue.

Due to the significant differences in GP and SP levels in BDEVs, we investigated the composition of the most abundant lipid classes and subclasses within these categories (Figure 3). For the GP category, this included PC, PE and PS lipid-subclasses (i.e., diacyl, alkylether $(\mathrm{O})$, plamalogen $(\mathrm{P})$, lysoacyl (L), lysoether (L-O) and lysoplasmalogen (L-P)) (Figures 3 and Supplementary 4A-C) and PS lipids at 'sum-composition' levels of annotation (Figures 3D and Supplementary 4D). At the GP-subclass level of annotation, common features of BDEVs were apparent including significant increases in mol\% diacyl-PC, diacyl-PE, and PS-O and PS-P lipids, and corresponding decreases in mol\% PC-O, acyl-LPE, diacyl-PS and LPS-O species relative to tissue (Figures 3A-C and Supplementary 4A-C). For the PS lipids, at the mol\% 'sum-composition' level of annotation, BDEVs exhibited extensive remodelling with significant increases in PS(O-37:6), PS(O-37:5), PS(O-37:4), PS(O-39:4), PS(39:5), PS(P-36:4), PS(P38:6), PS(P-38:5) and PS(P-38:4) and decreases in LPS(O-18:2), PS(36:2), PS(36:1) and PS(40:6) relative to tissue, irrespective of diagnosis (Figure 3D and Supplementary 4D). The observed increase in plasmalogen containing PS lipids is consistent with the results observed from the mol\% lipid subclass level of analysis that suggest dramatic remodelling of BDEV composition to incorporate more alkyl- and alkenyl-containing PS species rather than the more typical diacyl-containing species.

The frontal cortex contains a complex mixture of lipids coming from a multitude of cellular locations (plasma membrane, organelles and myelin sheath for example). By enriching for EVs from this tissue, we aimed to reduce the complexity of lipid profile and the signal to noise, to enable detection of changes in $\mathrm{AD}$ that were otherwise missed by profiling the gross tissue. Comparing CTL and AD BDEV at the category level (Figure $4 \mathrm{~A})$ revealed significant decrease in GP $(P<0.001)$ with a corresponding increase in SP in AD BDEVs relative to CTL BDEVs $(P<0.001)$ (mol\%, Figure $4 \mathrm{~A}$ and pmol/ $\mu \mathrm{g}$ protein, Supplemental Table 3).

Comparing at the subclass level revealed an approximately two-fold increase in PE-P (mol\%), accompanied with a significant decline in diacyl-PE, in AD BDEVs relative to CTL BDEVs (Figure 4B). At the PE mol\% 'sum-composition' level, significant acyl chain remodelling was observed in AD BDEVs, enabling differentiation from CTL samples. These included increased LPE(18:1), $\mathrm{PE}(\mathrm{P}-36: 2)$ and $\mathrm{PE}(\mathrm{P}-38: 4)$, and decreased $\operatorname{LPE}(22: 6), \operatorname{LPE}(22: 4), \operatorname{PE}(38: 4)$ and $\mathrm{PE}(40: 6)$ (Figure 4C). While no difference was observed between CTL vs AD BDEV in PC and PS subclasses (Supplemental Figures 5A and 5C), significant increases in PC(34:1), LPS(O-18:2) and PS(26:1), with corresponding decreases in PC(34:0), PC(36:1), PC(38:4) and PS(40:6) species were observed in 


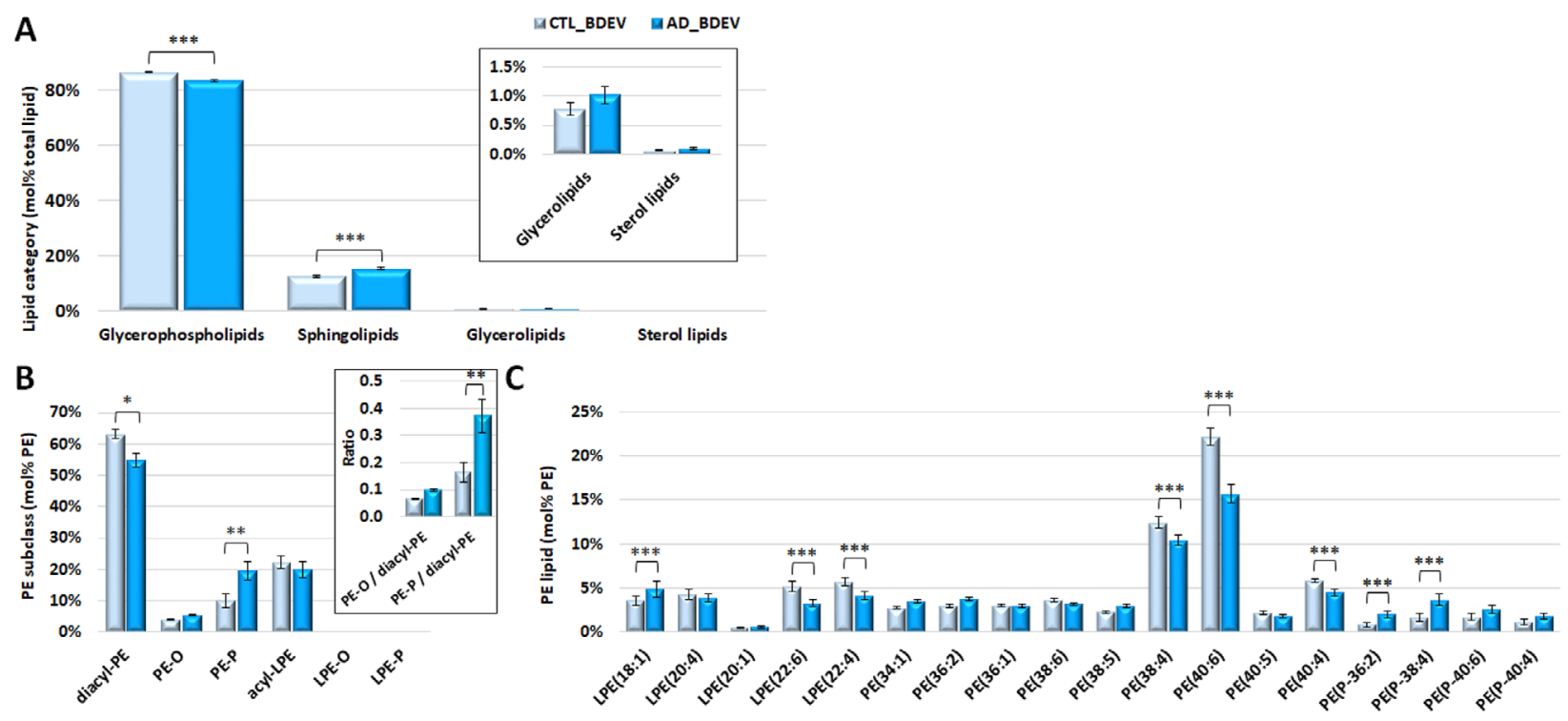

F I G U R E 4 Comparison of mol\% total lipid abundance differences between BDEV from control versus Alzheimer's disease tissue. [(A) Mol\% total lipid abundance distributions at the lipid category level. Four lipid categories, covering glycerophospholipids (GPs), sphingolipids (SPs), glycerolipids (GLs) and sterol lipids (STs) were included in this study. The inset shows the low abundant GL and ST categories for clarity. GPs were significantly decreased in AD vs. CTL BDEV, with a corresponding significant increase in SPs. (B) Mol\% total PE lipid subclass abundance distributions. Diacyl-PE was decreased and $\mathrm{PE}-\mathrm{P}$ was increased in AD BDEV. The inset shows the ratio of PE-O and PE-P to diacyl PE and a significant shift from diacyl-PE towards PE-P in AD. (C) Mol\% total PE lipid abundance distributions of individual PE molecules. LPE(18:1) was increased in AD relative to CTL. A group of polyunsaturated fatty acid (PUFA) containing PE molecules, including LPE(22:6), LPE(22:4), PE(38:4), PE(40:6) and PE(40:4), were significantly decreased in AD. A group of the most abundant PE-P lipids, including PE(P-36:2) and PE(P-38:4), was significantly increased in AD. Only the most abundant lipid molecules in each lipid class are shown for clarity. Data represent the average mol\% total lipid class abundance \pm standard error of the mean. Statistical significance was determined by ANOVA followed by Sidak's multiple comparison test, with multiplicity adjusted $P$ value $<0.01$. Adjusted $P$ value $<0.01$, ${ }^{* *}$ adjusted $P$ value $<0.001$, and ${ }^{* * *}$ adjusted $P$ value $<0.0001$. CTL, control; AD, Alzheimer's disease; BDEV, brain derived extracellular vesicles. $\mathrm{N}=8 \mathrm{AD}$ subjects and $\mathrm{N}=8 \mathrm{CTL}$ subjects]

AD vs. CTL BDEV (Supplemental Figure 5B and 5D). The PE lipid subclass and 'sum-composition' levels detected was also normalized based on absolute i.e. pmol/ $\mu \mathrm{g}$ protein abundances (Supplemental Figures 6 ) and based on lipidome wide normalization, i.e. mol\% total lipid (Supplemental Figures 7). All methods of normalization revealed a similar result. AD BDEVs contained at least half the amount of LPE(22:6), PE (38:4), PE(40:6) and PE(40:4) relative to CTL BDEVs (pmol/ug protein, $P<0.0001$ ) (Supplemental Figure 6).

The observed significant decrease in lipid species in AD BDEVs containing docosahexaenoic acid (C22:6 $\omega 3$ ) e.g., LPE(22:6), $\mathrm{PE}(40: 6)$ and PS(40:6), docosatetraenoic acid (C22:4 $\omega 6)$ e.g., LPE(22:4) and arachidonic acid (C20:4 $\omega 6)$ e.g., PC(38:4) and $\mathrm{PE}(38: 4)$ (each confirmed by HCD-MS/MS) (Figures 4C and Supplemental Figures 6 and 7), is consistent with overall remodelling of the lipid compositions to those that contain lower concentrations of polyunsaturated fatty acyl chains.

Comparison of the mol\% abundance of individual SM and Cer lipid species in AD BDEVs (Figures 5A and 5B, respectively) revealed significant decreases in $\mathrm{SM}(\mathrm{d} 36: 1), \mathrm{SM}(\mathrm{d} 38: 1)$ and $\operatorname{Cer}(\mathrm{d} 36: 1)$ species, and a corresponding increase in the very long chain containing SM(d42:2) (predominantly SM (d18:1/24:1)). This was offset by a significant decrease in the mol\% abundance of the sulfatide lipid sulfatide(d42:2) containing the same acyl chain composition (Supplemental Figure 8C), suggesting that additional remodelling has occurred within the galactosylceramide pathway of sphingolipid metabolism in AD BDEVs.

Taken together, these findings demonstrate that $\mathrm{AD}$ BDEV s have a unique lipid signature that distinguishes them from BDEVs of CTL frontal cortex, and highlights the utility of analysing EVs over gross tissue for the purposes of discovering disease related changes in lipid profile.

\section{4 | DISCUSSION}

Using optimized methods for the enrichment of BDEVs, coupled with deuterated internal standards and direct infusion nanoelectrospray ionization (nESI) and ultrahigh resolution and accurate mass spectrometry (UHRAMS) analysis (Rustam \& Reid, 2018; Wang et al., 2017), this study provides the first semi-quantitative characterization of the lipid composition of BDEVs derived from CNS tissue. In addition to comprising a lipid signature similar to EVs sourced from other biological materials, frontal cortexderived EVs were enriched in ether-containing PS lipids, a novel finding with implications for EV structure and function. The 
A

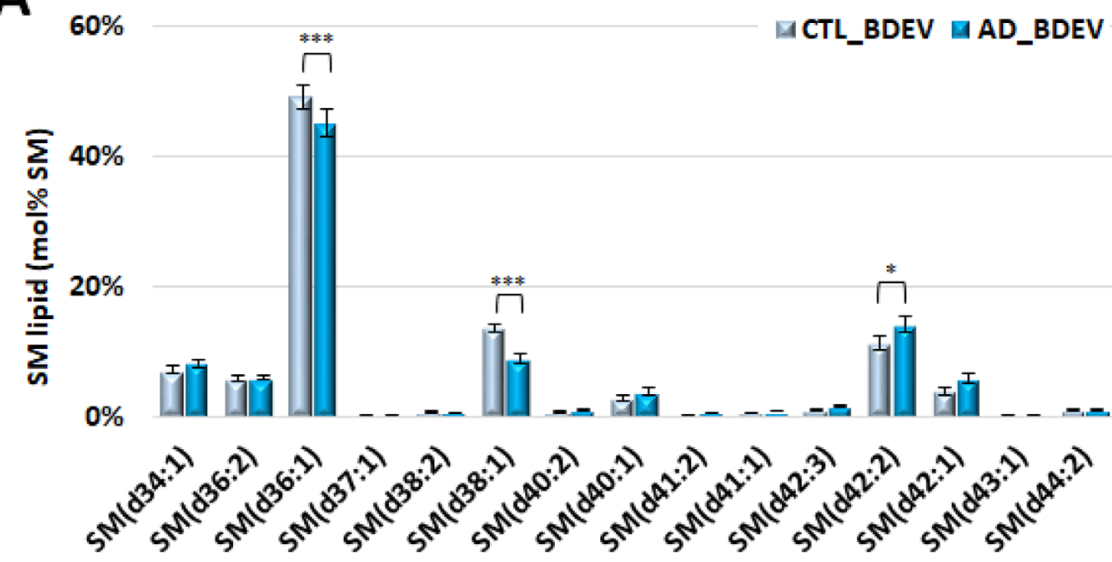

B

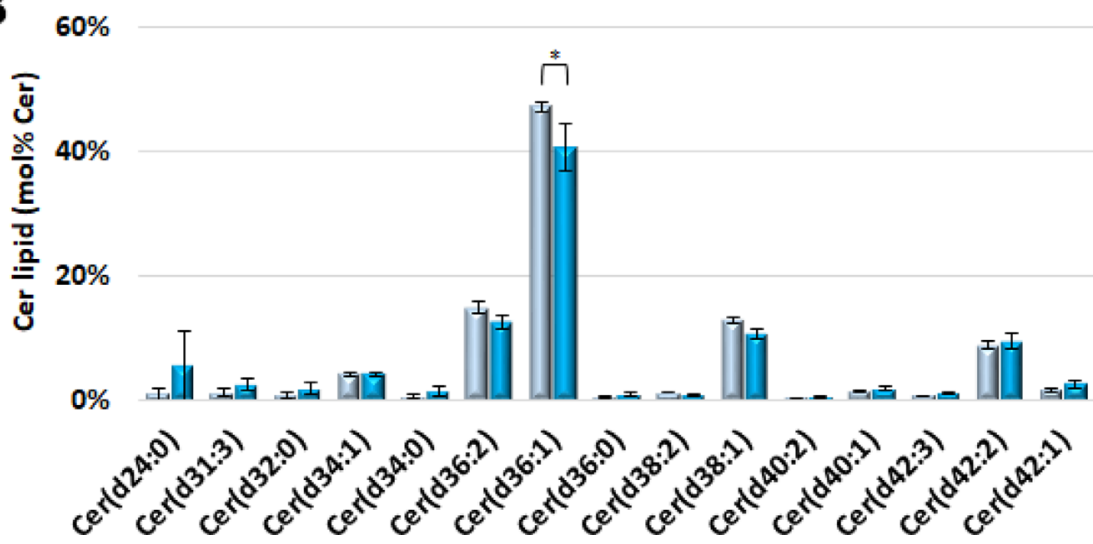

F I G U R E 5 Comparison of SM and Cer individual lipid molecules (mol\% class) of BDEV from control versus Alzheimer's disease tissue. [(A) Mol\% total SM lipid class abundance distributions. Significant decreases in $\operatorname{SM}(d 36: 1)$ and $\mathrm{SM}(\mathrm{d} 38: 1)$, accompanied with an increase in SM(d42:2), predominantly the SM(d18:1_24:1) species, were observed in $\mathrm{AD}$ vs. CTL BDEV. (B) Mol\% total Cer lipid class abundance distributions. Cer(d36:1) was found significantly lower in $\mathrm{AD}$ vs. CTL BDEV. Only the most abundant lipid molecules in each lipid class are shown for clarity. Data represent the average mol\% total lipid class abundance \pm standard error of the mean. Statistical significance was determined by ANOVA followed by Sidak's multiple comparison test, with multiplicity adjusted $P$ value $<0.01$. * Adjusted $P$ value $<0.01$, ${ }^{* *}$ adjusted $P$ value $<$ 0.001 , and ${ }^{* * *}$ adjusted $P$ value $<0.0001$. $\mathrm{CTL}$, control; AD, Alzheimer's disease; BDEV, brain derived extracellular vesicles. $\mathrm{N}=8$ $\mathrm{AD}$ subjects and $\mathrm{N}=8 \mathrm{CTL}$ subjects]

utility of BDEVs as a sensitive tool for the detection of lipid dyshomeostasis was highlighted, with significant remodelling of the frontal cortex lipidome revealed in BDEVs in $\mathrm{AD}$ in the absence of detectable changes in the parent tissue.

The lipid profiles of EVs have been reported for several cell lines and parasites, and from biological fluids including urine, plasma and serum (Brouwers et al., 2013; Haraszti et al., 2016; Laulagnier et al., 2004; Llorente et al., 2013; Lydic et al., 2015; Simbari et al., 2016; Skotland et al., 2017a, 2020; Sun et al., 2019). In agreement with these studies (Chen et al., 2019; Haraszti et al., 2016; Laulagnier et al., 2004; Llorente et al., 2013; Lydic et al., 2015; Skotland et al., 2017a, 2017b; Trajkovic et al., 2008), we show that human frontal cortex derived EVs are enriched in PS lipids. PS lipids make up a substantial proportion of the EV membrane and are proposed to play a role in facilitating EV uptake by recipient cells (Kastelowitz \& Yin, 2014; Laulagnier et al., 2004; Matsumura et al., 2019; Record et al., 2014, 2018; Sharma et al., 2017; Wei et al., 2016). Decreased diacyl-PC and higher levels of lysophospholipids, SM, Cer and ganglioside have previously been reported in EVs from urine, cell lines and blood (Haraszti et al., 2016; Llorente et al., 2013; Lydic et al., 2015; Skotland et al., 2017a, 2020; Sun et al., 2019). These findings were not observed here, likely due to the differences in cell and tissue type between studies.

While PC and PE ethers have been identified previously in EVs, here we reveal that EVs are abundant in alkyl- and alkenyl- (i.e., plasmalogen) ether-containing PS species, supporting the suggestion that ether lipids are a feature of EVs (Llorente et al., 2013; Lydic et al., 2015; Simbari et al., 2016; Skotland et al., 2017a, 2019). Ether lipids can modulate membrane rigidity and permeability (Dorninger et al., 2017; Glaser \& Gross, 1994; Wallner \& Schmitz, 2011), possibly mediating EV interaction with host cells and contributing to stability in the environment.

Lipid remodelling, specifically alterations in GP and SP, are associated with AD pathogenesis (Bennett et al., 2013; Han et al., 2011; He et al., 2010; Kosicek \& Hecimovic, 2013; Mielke \& Lyketsos, 2010; Mielke et al., 2010a, 2010b, 2011, 2014; Wong et al., 2017; Wood, 2012). The relative levels of these lipids reported in previous studies however are partially conflicting, likely due to the differences in the brain region examined, disease severity, comorbidity and detection methods (Bandaru et al., 2009; Cutler et al., 2004; Han et al., 2001, 2002; He et al., 2010; Kosicek \& Hecimovic, 2013; Pettegrew et al., 2001; Wong et al., 2017; Wood, 2012).

Down regulation of the glycerophospholipids PC, PE and glycerophosphatidylinositol (PI) in AD, with a general decline in plasmalogen lipids, mainly PC-P and PE-P, has been observed in multiple brain regions in AD (Ginsberg et al., 1995; Grimm et al., 2011; Han et al., 2001; Igarashi et al., 2011; Kosicek \& Hecimovic, 2013; Wood, 2012). No significant changes were detected in plasmalogen levels in AD frontal cortex (relative to CTL) in this study, however clear differences in ether-PE lipids, including both PE-O and PE-P, were observed in the BDEVs isolated from these tissues. Specifically, plasmalogen PE molecules 
$\mathrm{PE}(\mathrm{P}-36: 2)$ and $\mathrm{PE}(\mathrm{P}-38: 4)$, were significantly increased in BDEV in AD. Plasmalogens are abundant in the CNS and are considered an antioxidant due to the presence of a carbon-carbon double bond at the ether linkage that is prone to oxidation (Dorninger et al., 2017; Su et al., 2019; Wallner \& Schmitz, 2011). Plasmalogens could play an active role in scavenging oxidative stress and reducing inflammatory responses in recipient cells with studies showing that PE-P can rescue neuronal cell death (Hossain et al., 2013; Wood et al., 2010). Whether donor cells become more susceptible to oxidation following release of PE-P via BDEVs is not yet known, with further studies required to determine their role in disease.

The CNS is highly enriched with poly unsaturated fatty acid (PUFA), with the majority of the PUFA content encapsulated in GP (Bazinet \& Laye, 2014; Dyall, 2015). The BDEVs in this study recapitulated this brain-specific feature. Moreover, the C22:6 (i.e., docosahexaenoic acid, DHA) fatty acyl chain-containing species LPE(22:6), PE(40:6) and PS(40:6) were found to be decreased $\sim$ two fold in AD BDEVs. Notably, DHA is the most abundant PUFA in GP, and DHA-derived bioactive anti-inflammatory specialised pro-resolving mediators (SPM) (Serhan \& Levy, 2018; Whittington et al., 2017) are capable of attenuating Aß amyloidogenesis and enhancing $A ß$ phagocytosis in AD (Fiala et al., 2015; Lukiw et al., 2005). The decreased C22:6 content in AD BDEVs in this study supports DHA deficiency in AD, a known feature of the disease (Morris et al., 2003; Tully et al., 2003) and suggest that the level of C22:6 in BDEVs could serve as a peripheral marker of AD.

As sphingolipid metabolism has also been reported to be remodelled in AD (Haughey et al., 2010; He et al., 2010; Mielke \& Lyketsos, 2010; Wood, 2012), we investigated lipid profile changes in SM and Cer lipids. SM is predominantly found on the plasma membrane and is one of the main components of lipid-rafts. The abundance of SM lipids appear to be modified in AD, however there is some contention as to whether SM is decreased or increased, with reports suggesting variability between brain regions (Bandaru et al., 2009; Chan et al., 2012; Cutler et al., 2004; He et al., 2010; Kosicek \& Hecimovic, 2013; Pettegrew et al., 2001). SM(d36:1) and SM(d38:1) were decreased in AD BDEVs, accompanied by an increase in very long chain SM (d42:2), predominantly the SM(d18:1_24:1) species. It is unclear from our study, the implications APOE4 (risk factor for AD) genotype has on SM levels in EVs. Given the role APOE has on lipid transport, future investigations aimed at dissecting the contribution of APOE genotype to EV composition are warranted.

With the knowledge that lipid metabolism is altered in AD in the CNS, researchers have examined the lipidome of CSF and blood in the search for potential biomarkers, with varied results (Han et al., 2003; Koal et al., 2015; Kosicek et al., 2012; Mielke et al., 2010a, 2010b, 2011, 2014). Anand et al. (2017) discovered that LPC and a group of lipid peroxidation products, including oxidized PC, oxidized-triacylglyceride (TG) and F2-isoprostanes, were up-regulated, while PC, SM, PE, especially PE-P, were found to be declined in AD serum (Barupal et al., 2019; Gonzalez-Dominguez et al., 2014; Goodenowe et al., 2007). PUFA PC species (e.g., PC(16:0/20:5), PC(16:0/22:6), PC(18:0/22:6) etc.) have been reported to be decreased in preclinical AD plasma (Fiandaca et al., 2015; Mapstone et al., 2014; Whiley et al., 2014). The major drawback of these approaches relates to the complexity of the lipidome and measurement of changes not necessarily specific to the CNS or disease which reduces diagnostic accuracy and reproducibility. Other diseases, or co-morbidities such as diabetes and cardiovascular disease, can also contribute to the alteration of the lipid profile, further complicating lipid biomarker discovery.

An ideal lipid peripheral biomarker would ideally be brain-derived to reflect early changes in the CNS in AD. BDEVs can readily cross the blood brain barrier (BBB) (Kanninen et al., 2016; Saeedi et al., 2019; Skoumalová et al., 2011), enabling the study of BDEV lipids in the periphery, effectively eliminating many of the caveats associated with studying complex fluids such as serum or plasma. Our research suggests that future studies should assess ether PE lipids and polyunsaturated fatty acyl containing lipids in peripherally-sourced BDEVs alongside other clinical measures (CSF and neuroimaging assessments) to determine if BDEVs in the periphery can predict progression from mild cognitive impairment (MCI) to AD.

\section{ACKNOWLEDGEMENTS}

This work was supported by grants from the Australian National Health and Medical Research Council (GNT1041413 and GNT1002349 to AFH; 628946 to CLM, KJB, and AFH), the Australian Research Council (LE160100015 to GER), the Bethlehem Griffiths Research Foundation to LJV (Australia), the Alzheimer's Australia Dementia Research Foundation John Shutes Project Grant to LJV and The Alzheimer's Association (AARF-18-566256) to LJV (U.S.A). We thank Fairlie Hinton and Geoffrey Pavey from the Victorian Brain Bank.

\section{REFERENCES}

Anand, S., Barnes, J. M., Young, S. A., Garcia, D. M., Tolley, H. D., Kauwe, J. S. K., \& Graves, S. W. (2017). Discovery and confirmation of diagnostic serum lipid biomarkers for Alzheimer's disease using direct infusion mass spectrometry. Journal of Alzheimer's Disease, 59, 277-290.

Bandaru, V. V., Troncoso, J., Wheeler, D., Pletnikova, O., Wang, J., Conant, K., \& Haughey, N. J. (2009). ApoE4 disrupts sterol and sphingolipid metabolism in Alzheimer's but not normal brain. Neurobiology of Aging, 30, 591-599.

Barupal, D. K., Baillie, R., Fan, S., Saykin, A. J., Meikle, P. J., Arnold, M., Nho, K., Fiehn, O., Kaddurah-DAOUK, R., \& Alzheimer Disease Metabolomics, C. (2019). Sets of coregulated serum lipids are associated with Alzheimer's disease pathophysiology. Alzheimers \& Dementia, 11, 619-627.

Bazinet, R. P., \& Laye, S. (2014). Polyunsaturated fatty acids and their metabolites in brain function and disease. Nature Reviews Neuroscience, 15, 771-785.

Bennett, S. A., Valenzuela, N., Xu, H., Franko, B., Fai, S., \& Figeys, D. (2013). Using neurolipidomics to identify phospholipid mediators of synaptic (dys)function in Alzheimer's Disease. Front Physiol, 4. 
Brouwers, J. F., Aalberts, M., Jansen, J. W., Van Niel, G., Wauben, M. H., Stout, T. A., Helms, J. B., \& Stoorvogel, W. (2013). Distinct lipid compositions of two types of human prostasomes. Proteomics, 13, 1660-1666.

Chan, R. B., Oliveira, T. G., Cortes, E. P., Honig, L. S., Duff, K. E., Small, S. A., Wenk, M. R., SHUI, G., \& Di PAOLO, G. (2012). Comparative lipidomic analysis of mouse and human brain with Alzheimer disease. Journal of Biological Chemistry, 287, 2678-2688.

Chen, S., Datta-Chaudhuri, A., Deme, P., Dickens, A., Dastgheyb, R., Bhargava, P., Bi, H., \& Haughey, N. J. (2019). Lipidomic characterization of extracellular vesicles in human serum. Journal of Circulating Biomarkers, 8, 1849454419879848.

Cheng, L., Vella, L. J., Barnham, K. J., Mclean, C., Masters, C. L., \& Hill, A. F. (2020). Small RNA fingerprinting of Alzheimer's disease frontal cortex extracellular vesicles and their comparison with peripheral extracellular vesicles. Journal of Extracellular Vesicles, 9, 1766822.

Cutler, R. G., Kelly, J., Storie, K., Pedersen, W. A., Tammara, A., Hatanpaa, K., Troncoso, J. C., \& Mattson, M. P. (2004). Involvement of oxidative stress-induced abnormalities in ceramide and cholesterol metabolism in brain aging and Alzheimer's disease. Proceedings of the National Academy of Sciences, 101, $2070-2075$.

Deleo, A. M., \& Ikezu, T. (2018). Extracellular vesicle biology in Alzheimer's disease and related tauopathy. Journal of Neuroimmune Pharmacology, 13, $292-308$.

Dorninger, F., Forss-Petter, S., \& Berger, J. (2017). From peroxisomal disorders to common neurodegenerative diseases - the role of ether phospholipids in the nervous system. Febs Letters, 591, 2761-2788.

Dyall, S. C. (2015). Long-chain omega-3 fatty acids and the brain: A review of the independent and shared effects of EPA, DPA and DHA. Frontiers in Aging Neuroscience, 7

Fahy, E., Subramaniam, S., Brown, H. A., Glass, C. K., Merrill, A. H., Murphy, R. C., Raetz, C. R. H., Russell, D. W., Seyama, Y., Shaw, W., Shimizu, T., Spener, F., Van Meer, G., Vannieuwenhze, M. S., White, S. H., Witztum, J. L., \& Dennis, E. A. (2005). A comprehensive classification system for lipids. Journal of Lipid Research, 46, 839-862.

Fiala, M., Halder, R. C., Sagong, B., Ross, O., Sayre, J., Porter, V., \& Bredesen, D. E. (2015). Omega-3 supplementation increases amyloid-beta phagocytosis and resolvin D1 in patients with minor cognitive impairment. Faseb Journal, 29, 2681-2689.

Fiandaca, M. S., Zhong, X., Cheema, A. K., Orquiza, M. H., Chidambaram, S., Tan, M. T., Gresenz, C. R., Fitzgerald, K. T., Nalls, M. A., Singleton, A. B., Mapstone, M., \& Federoff, H. J. (2015). Plasma 24-metabolite panel predicts preclinical transition to clinical stages of Alzheimer's disease. Frontiers in Neurology, 6.

Ginsberg, L., Rafique, S., Xuereb, J. H., Rapoport, S. I., \& Gershfeld, N. L. (1995). Disease and anatomic specificity of ethanolamine plasmalogen deficiency in Alzheimer's disease brain. Brain Research, 698, 223-226.

Glaser, P. E., \& Gross, R. W. (1994). Plasmenylethanolamine facilitates rapid membrane fusion: A stopped-flow kinetic investigation correlating the propensity of a major plasma membrane constituent to adopt an HII phase with its ability to promote membrane. Biochemistry, 33, 5805-5812.

Gonzalez-Dominguez, R., Garcia-Barrera, T., \& Gomez-Ariza, J. L. (2014). Using direct infusion mass spectrometry for serum metabolomics in Alzheimer's disease. Analytical and Bioanalytical Chemistry, 406, 7137-7148.

Goodenowe, D. B., Cook, L. L., Liu, J., Lu, Y., Jayasinghe, D. A., Ahiahonu, P. W. K., Heath, D., Yamazaki, Y., Flax, J., Krenitsky, K. F., Sparks, D. L., Lerner, A., Friedland, R. P., Kudo, T., Kamino, K., Morihara, T., Takeda, M., \& Wood, P. L. (2007). Peripheral ethanolamine plasmalogen deficiency: A logical causative factor in Alzheimer's disease and dementia. Journal of Lipid Research, 48, 2485-2498.

Grimm, M. O., Kuchenbecker, J., Rothhaar, T. L., Grosgen, S., Hundsdorfer, B., Burg, V. K., Friess, P., Muller, U., Grimm, H. S., Riemenschneider, M., \& Hartmann, T. (2011). Plasmalogen synthesis is regulated via alkyl-dihydroxyacetonephosphate-synthase by amyloid precursor protein processing and is affected in Alzheimer's disease. Journal of Neurochemistry, 116, 916-925.

Han, X., Fagan, A. M., Cheng, H., Morris, J. C., Xiong, C., \& Holtzman, D. M. (2003). Cerebrospinal fluid sulfatide is decreased in subjects with incipient dementia. Annals of Neurology, 54, 115-119.

Han, X., Holtzman, D. M., \& Mckeel, D. M. (2001). Plasmalogen deficiency in early Alzheimer's disease subjects and in animal models: Molecular characterization using electrospray ionization mass spectrometry. Journal of Neurochemistry, 77, 1168-1180.

Han, X., Holtzman, D. M., Mckeel, D. M., Kelley, J., \& Morris, J. C. (2002). Substantial sulfatide deficiency and ceramide elevation in very early Alzheimer's disease: Potential role in disease pathogenesis. Journal of Neurochemistry, 82, 809-818.

Han, X., Rozen, S., Boyle, S. H., Hellegers, C., Cheng, H., Burke, J. R., Welsh-Bohmer, K. A., Doraiswamy, P. M., \& Kaddurah-Daouk, R. (2011). Metabolomics in early Alzheimer's disease: Identification of altered plasma sphingolipidome using shotgun lipidomics. Plos One, 6, e21643.

Haraszti, R. A., Didiot, M. C., Sapp, E., Leszyk, J., Shaffer, S. A., Rockwell, H. E., Gao, F., Narain, N. R., Difiglia, M., Kiebish, M. A., Aronin, N., \& Khvorova, A. (2016). High-resolution proteomic and lipidomic analysis of exosomes and microvesicles from different cell sources. Journal of Extracellular Vesicles, 5, 32570.

Haughey, N. J., Bandaru, V. V. R., Bae, M., \& Mattson, M. P. (2010). Roles for dysfunctional sphingolipid metabolism in Alzheimer's disease neuropathogenesis. Biochimica et Biophysica Acta (BBA) - Molecular and Cell Biology of Lipids, 1801, 878-886.

He, X., Huang, Y., Li, B., Gong, C.-X., \& Schuchman, E. H. (2010). Deregulation of sphingolipid metabolism in Alzheimer's disease. Neurobiology of Aging, 31, 398-408.

Hessvik, N. P., \& Llorente, A. (2017). Current knowledge on exosome biogenesis and release. Cellular and Molecular Life Sciences, 75, $193-208$.

Hossain, M. S., Ifuku, M., Take, S., Kawamura, J., Miake, K., \& Katafuchi, T. (2013). Plasmalogens rescue neuronal cell death through an activation of AKT and ERK survival signaling. Plos One, 8, e83508.

Igarashi, M., Ma, K., Gao, F., Kim, H. W., Rapoport, S. I., \& Rao, J. S. (2011). Disturbed choline plasmalogen and phospholipid fatty acid concentrations in Alzheimer's disease prefrontal cortex. Journal of Alzheimers Disease, 24, 507-517.

Johnstone, R. M. A., Adam M., Hammond, J. R., Orr, L., \& Turbide, C. (1987). Vesicle formation during reticulocyte maturation. The Journal of Biological Chemistry, 262, 9412-9420.

Kanninen, K. M., Bister, N., Koistinaho, J., \& Malm, T. (2016). Exosomes as new diagnostic tools in CNS diseases. Biochimica et Biophysica Acta (BBA) - Molecular Basis of Disease, 1862, 403-410.

Kastelowitz, N., \& Yin, H. (2014). Exosomes and microvesicles: Identification and targeting by particle size and lipid chemical probes. Chembiochem, 15, 923-928.

Koal, T., Klavins, K., Seppi, D., Kemmler, G., \& Humpel, C. (2015). Sphingomyelin SM(d18:1/18:0) is significantly enhanced in cerebrospinal fluid samples dichotomized by pathological amyloid-beta42, tau, and phospho-tau-181 levels. Journal of Alzheimers Disease, 44, 1193-1201.

Kosicek, M., \& Hecimovic, S. (2013). Phospholipids and Alzheimer's disease: Alterations, mechanisms and potential biomarkers. International Journal of Molecular Sciences, 14, 1310-1322.

Kosicek, M., Zetterberg, H., Andreasen, N., Peter-Katalinic, J., \& Hecimovic, S. (2012). Elevated cerebrospinal fluid sphingomyelin levels in prodromal Alzheimer's disease. Neuroscience Letters, 516, 302-305.

Laulagnier, K., Motta, C., Hamdi, S., Roy, S., Fauvelle, F., Pageaux, J., Kobayashi, T., Salles, J., Perret, B., Bonnerot, C., \& Record, M. (2004). Mast cell- and dendritic cell-derived exosomes display a specific lipid composition and an unusual membrane organization. The Biochemical Journal, 380 , 161-171.

Li, W., Li, C., Zhou, T., Liu, X., Liu, X., Li, X., \& Chen, D. (2017). Role of exosomal proteins in cancer diagnosis. Molecular Cancer, 16(1). 
Llorente, A., Skotland, T., Sylvänne, T., Kauhanen, D., Róg, T., Orłowski, A., Vattulainen, I., Ekroos, K., \& Sandvig, K. (2013). Molecular lipidomics of exosomes released by PC-3 prostate cancer cells. Biochimica et Biophysica Acta (BBA) - Molecular and Cell Biology of Lipids, 1831, 1302-1309.

Lukiw, W. J., Cui, J. G., Marcheselli, V. L., Bodker, M., Botkjaer, A., Gotlinger, K., Serhan, C. N., \& Bazan, N. G. (2005). A role for docosahexaenoic acid-derived neuroprotectin D1 in neural cell survival and Alzheimer disease. Journal of Clinical Investigation, 115, 2774-2783.

Lydic, T. A., Townsend, S., Adda, C. G., Collins, C., Mathivanan, S., \& Reid, G. E. (2015). Rapid and comprehensive 'shotgun' lipidome profiling of colorectal cancer cell derived exosomes. Methods (San Diego, Calif.), 87, 83-95.

Mapstone, M., Cheema, A. K., Fiandaca, M. S., Zhong, X., Mhyre, T. R., Macarthur, L. H., Hall, W. J., Fisher, S. G., Peterson, D. R., Haley, J. M., Nazar, M. D., Rich, S. A., Berlau, D. J., Peltz, C. B., Tan, M. T., Kawas, C. H., \& Federoff, H. J. (2014). Plasma phospholipids identify antecedent memory impairment in older adults. Nature Medicine, 20, 415-418.

Matsumura, S., Minamisawa, T., Suga, K., Kishita, H., Akagi, T., Ichiki, T., Ichikawa, Y., \& Shiba, K. (2019). Subtypes of tumour cell-derived small extracellular vesicles having differently externalized phosphatidylserine. Journal of Extracellular Vesicles, 8, 1579541.

Melo, S. A., Luecke, L. B., Kahlert, C., Fernandez, A. F., Gammon, S. T., Kaye, J., Lebleu, V. S., Mittendorf, E. A., Weitz, J., Rahbari, N., Reissfelder, C., Pilarsky, C., Fraga, M. F., Piwnica-Worms, D., \& Kalluri, R. (2015). Glypican-1 identifies cancer exosomes and detects early pancreatic cancer. Nature, 523, 177-182.

Mielke, M. M., Bandaru, V. V. R., Haughey, N. J., Rabins, P. V., Lyketsos, C. G., \& Carlson, M. C. (2010a). Serum sphingomyelins and ceramides are early predictors of memory impairment. Neurobiology of Aging, 31, 17-24.

Mielke, M. M., Haughey, N. J., Bandaru, V. V., Schech, S., Carrick, R., Carlson, M. C., Mori, S., Miller, M. I., Ceritoglu, C., Brown, T., Albert, M., \& Lyketsos, C. G. (2010b). Plasma ceramides are altered in mild cognitive impairment and predict cognitive decline and hippocampal volume loss. Alzheimers \& Dementia, 6, 378-385.

Mielke, M. M., Haughey, N. J., Bandaru, V. V. R., Weinberg, D. D., Darby, E., Zaidi, N., Pavlik, V., Doody, R. S., \& Lyketsos, C. G. (2011). Plasma sphingomyelins are associated with cognitive progression in Alzheimer's disease. Journal of Alzheimer's Disease, 27, 259-269.

Mielke, M. M., Haughey, N. J., Bandaru, V. V. R., Zetterberg, H., Blennow, K., Andreasson, U., Johnson, S. C., Gleason, C. E., Blazel, H. M., Puglielli, L., Sager, M. A., Asthana, S., \& Carlsson, C. M. (2014). Cerebrospinal fluid sphingolipids, beta-amyloid, and tau in adults at risk for Alzheimer's disease. Neurobiology of Aging, 35, 2486-2494.

Mielke, M. M., \& Lyketsos, C. G. (2010). Alterations of the sphingolipid pathway in Alzheimer's disease: New biomarkers and treatment targets? Neuromolecular Medicine, 12, 331-340.

Morris, M. C., Evans, D. A., Bienias, J. L., Tangney, C. C., Bennett, D. A., Wilson, R. S., Aggarwal, N., \& Schneider, J. (2003). Consumption of fish and n-3 fatty acids and risk of incident Alzheimer Disease. Archives of Neurology, 60, 940-946.

Pettegrew, J. W., Panchalingam, K., Hamilton, R. L., \& Mcclure, R. J. (2001). Brain membrane phospholipid alterations in Alzheimer's disease. Neurochemical Research, 26, 771-782.

Phuyal, S., Skotland, T., Hessvik, N. P., Simolin, H., Overbye, A., Brech, A., Parton, R. G., Ekroos, K., Sandvig, K., \& Llorente, A. (2015). The ether lipid precursor hexadecylglycerol stimulates the release and changes the composition of exosomes derived from PC-3 cells. Journal of Biological Chemistry, 290, 4225-4237.

Rai, A., Greening, D. W., Chen, M., Xu, R., Ji, H., \& Simpson, R. J. (2019). Exosomes derived from human primary and metastatic colorectal cancer cells contribute to functional heterogeneity of activated fibroblasts by reprogramming their proteome. Proteomics, 19(8).

Rajendran, L., Honsho, M., Zahn, T. R., Keller, P., Geiger, K. D., Verkade, P., \& Simons, K. (2006). Alzheimer's disease beta-amyloid peptides are released in association with exosomes. Proceedings of the National Academy of Sciences, 103, 11172-11177.

Raposo, G., Nijman, H. W., Stoorvogel, W., Leijendekker, R., Harding, C. V. M., Cornelis, J. M., \& Geuze, H. J. (1996). B lymphocytes secrete antigen-presenting vesicles. Journal of Experimental Medicine, 183, 1161-1172.

Record, M., Carayon, K., Poirot, M., \& Silvente-Poirot, S. (2014). Exosomes as new vesicular lipid transporters involved in cell-cell communication and various pathophysiologies. Biochimica Et Biophysica Acta, 1841, 108-120.

Record, M., Silvente-Poirot, S., Poirot, M., \& Wakelam, M. J. O. (2018). Extracellular vesicles: Lipids as key components of their biogenesis and functions. Journal of Lipid Research, 59, 1316-1324.

Rustam, Y. H., \& Reid, G. E. (2018). Analytical challenges and recent advances in mass spectrometry based lipidomics. Analytical Chemistry, 90, 374-397.

Ryan, E., \& Reid, G. E. (2016). Chemical Derivatization and ultrahigh resolution and accurate mass spectrometry strategies for "shotgun" lipidome analysis. Accounts of Chemical Research, 49, 1596-1604.

Saeedi, S., Israel, S., Nagy, C., \& Turecki, G. (2019). The emerging role of exosomes in mental disorders. Translational Psychiatry, 9(1).

Satoi, H., Tomimoto, H., Ohtani, R., Kitano, T., Kondo, T., Watanabe, M., Oka, N., Akiguchi, I., Furuya, S., Hirabayashi, Y., \& Okazaki, T. (2005). Astroglial expression of ceramide in Alzheimer's disease brains: A role during neuronal apoptosis. Neuroscience, 130, 657-666.

Serhan, C. N., \& Levy, B. D. (2018). Resolvins in inflammation: Emergence of the pro-resolving superfamily of mediators. Journal of Clinical Investigation, 128, 2657-2669.

Serna, J., Garcia-Seisdedos, D., Alcazar, A., Lasuncion, M. A., Busto, R., \& Pastor, O. (2015). Quantitative lipidomic analysis of plasma and plasma lipoproteins using MALDI-TOF mass spectrometry. Chemistry and Physics of Lipids, 189, 7-18.

Sharma, R., Huang, X., Brekken, R. A., \& Schroit, A. J. (2017). Detection of phosphatidylserine-positive exosomes for the diagnosis of early-stage malignancies. British Journal of Cancer, 117, 545-552.

Sharples, R. A., Vella, L. J., Nisbet, R. M., Naylor, R., Perez, K., Barnham, K. J., Masters, C. L., \& Hill, A. F. (2008). Inhibition of gamma-secretase causes increased secretion of amyloid precursor protein C-terminal fragments in association with exosomes. Faseb Journal, 22, 1469-1478.

Simbari, F., Mccaskill, J., Coakley, G., Millar, M., Maizels, R. M., FabriÁS, G., Casas, J., \& Buck, A. H. (2016). Plasmalogen enrichment in exosomes secreted by a nematode parasite versus those derived from its mouse host: Implications for exosome stability and biology. Journal of Extracellular Vesicles, 5, 30741.

Simons, M., \& Raposo, G. (2009). Exosomes - vesicular carriers for intercellular communication. Current Opinion in Cell Biology, 21, 575-581.

Skotland, T., Ekroos, K., Kauhanen, D., Simolin, H., Seierstad, T., Berge, V., Sandvig, K., \& Llorente, A. (2017a). Molecular lipid species in urinary exosomes as potential prostate cancer biomarkers. European Journal of Cancer, 70, 122-132.

Skotland, T., Hessvik, N. P., Sandvig, K., \& Llorente, A. (2019). Exosomal lipid composition and the role of ether lipids and phosphoinositides in exosome biology. Journal of Lipid Research, 60, 9-18.

Skotland, T., Sagini, K., Sandvig, K., \& Llorente, A. (2020). An emerging focus on lipids in extracellular vesicles. Advanced Drug Delivery Reviews, 159, 308-321.

Skotland, T., Sandvig, K., \& Llorente, A. (2017b). Lipids in exosomes: Current knowledge and the way forward. Progress in Lipid Research, 66, 30-41.

Skoumalová, A., Ivica, J., Šantorová, P., Topinková, E., \& Wilhelm, J. (2011). The lipid peroxidation products as possible markers of Alzheimer's disease in blood. Experimental Gerontology, 46, 38-42.

Su, X. Q., Wang, J. M., \& Sinclair, A. J. (2019). Plasmalogens and Alzheimer's disease: A review. Lipids in Health and Disease, 18(1). 
Sun, Y., Saito, K., \& Saito, Y. (2019). Lipid profile characterization and lipoprotein comparison of extracellular vesicles from human plasma and serum. Metabolites, 9(11).

Théry, C., Witwer, K. W., Aikawa, E., Alcaraz, M. J., Anderson, J. D., Andriantsitohaina, R., Antoniou, A., Arab, T., Archer, F., Atkin-Smith, G. K., Ayre, D. C., Bach, J. M., Bachurski, D., Baharvand, H., Balaj, L., Baldacchino, S., Bauer, N. N., Baxter, A. A., Bebawy, M. ... Zuba-Surma, E. K. (2018). Minimal information for studies of extracellular vesicles 2018 (MISEV2018): A position statement of the International Society for Extracellular Vesicles and update of the MISEV2014 guidelines. Journal of Extracellular Vesicles, 7, 1535750.

Thompson, A. G., Gray, E., Heman-Ackah, S. M., Mager, I., Talbot, K., Andaloussi, S. E., Wood, M. J., \& Turner, M. R. (2016). Extracellular vesicles in neurodegenerative disease - pathogenesis to biomarkers. Nature Reviews Neurology, 12, 346-357.

Trajkovic, K., Hsu, C., Chiantia, S., Rajendran, L., Wenzel, D., Wieland, F., Schwille, P., Brügger, B., \& Simons, M. (2008). Ceramide triggers budding of exosome vesicles into multivesicular endosomes. Science, 319, 1244-1247.

Tully, A. M., Roche, H. M., Doyle, R., Fallon, C., Bruce, I., Lawlor, B., Coakley, D., \& Gibney, M. J. (2003). Low serum cholesteryl ester-docosahexaenoic acid levels in Alzheimer's disease: A case-control study. British Journal of Nutrition, 89, 483-489.

Vella, L. J., Hill, A. F., \& Cheng, L. (2016). Focus on extracellular vesicles: Exosomes and their role in protein trafficking and biomarker potential in Alzheimer's and Parkinson's disease. International Journal of Molecular Sciences, 17(2).

Vella, L. J., Scicluna, B. J., Cheng, L., Bawden, E. G., Masters, C. L., Ang, C. S., Willamson, N., Mclean, C., Barnham, K. J., \& Hill, A. F. (2017). A rigorous method to enrich for exosomes from brain tissue. Journal of Extracellular Vesicles, 6, 1348885

Vingtdeux, V., Sergeant, N., \& Buée, L. (2012). Potential contribution of exosomes to the prion-like propagation of lesions in Alzheimer's disease. Frontiers in Physiology, 3.

Wallner, S., \& Schmitz, G. (2011). Plasmalogens the neglected regulatory and scavenging lipid species. Chemistry and Physics of Lipids, 164, 573-589.

Wang, H., \& Eckel, R. H. (2014). What are lipoproteins doing in the brain? Trends in Endocrinology \& Metabolism, 25, 8-14.

Wang, M., Wang, C., \& Han, X. (2017). Selection of internal standards for accurate quantification of complex lipid species in biological extracts by electrospray ionization mass spectrometry-What, how and why? Mass Spectrometry Reviews, 36, 693-714.

Wei, X., Liu, C., Wang, H., Wang, L., Xiao, F., Guo, Z., \& Zhang, H. (2016). Surface phosphatidylserine Is responsible for the internalization on microvesicles derived from hypoxia-induced human bone marrow mesenchymal stem cells into human endothelial cells. Plos One, 11, e0147360.

Whiley, L., Sen, A., Heaton, J., Proitsi, P., García-Gómez, D., Leung, R., Smith, N., Thambisetty, M., Kloszewska, I., Mecocci, P., Soininen, H., Tsolaki, M., Vellas, B., Lovestone, S., \& Legido-Quigley, C. (2014). Evidence of altered phosphatidylcholine metabolism in Alzheimer's disease. Neurobiology of Aging, 35, 271-278.

Whittington, R. A., Planel, E., \& Terrando, N. (2017). Impaired resolution of inflammation in Alzheimer's disease: A review. Frontiers in immunology, 8.

Wong, M. W., Braidy, N., Poljak, A., Pickford, R., Thambisetty, M., \& Sachdev, P. S. (2017). Dysregulation of lipids in Alzheimer's disease and their role as potential biomarkers. Alzheimers \& Dementia, 13, 810-827.

Wood, P. L. (2012). Lipidomics of Alzheimer's disease: Current status. Alzheimer's Research \& Therapy, 4(1).

Wood, P. L., Mankidy, R., Ritchie, S., Heath, D., Wood, J. A., Flax, J., \& Goodenowe, D. B. (2010). Circulating plasmalogen levels and Alzheimer Disease Assessment Scale-Cognitive scores in Alzheimer patients. Journal of Psychiatry and Neuroscience, 35, 59-62.

\section{SUPPORTING INFORMATION}

Additional supporting information may be found online in the Supporting Information section at the end of the article.

How to cite this article: Su, H., Rustam, Y. H., Masters, C. L., Makalic, E., McLean, C., Hill, A. F., Barnham, K. J., Reid, G. E., \& Vella, L. J. (2021). Characterization of brain-derived extracellular vesicle lipids in Alzheimer's disease. Journal of Extracellular Vesicles, 10,e12089. https://doi.org/10.1002/jev2.12089 


\section{University Library}

\section{- M I N E R VA}

\section{A gateway to Melbourne's research publications}

Minerva Access is the Institutional Repository of The University of Melbourne

\section{Author/s:}

Su, H;Rustam, YH;Masters, CL;Makalic, E;McLean, CA;Hill, AF;Barnham, KJ;Reid, GE;Vella, LJ

Title:

Characterization of brain-derived extracellular vesicle lipids in Alzheimer's disease

Date:

2021-05-01

\section{Citation:}

Su, H., Rustam, Y. H., Masters, C. L., Makalic, E., McLean, C. A., Hill, A. F., Barnham, K. J., Reid, G. E. \& Vella, L. J. (2021). Characterization of brain-derived extracellular vesicle lipids in Alzheimer's disease. JOURNAL OF EXTRACELLULAR VESICLES, 10 (7), https:// doi.org/10.1002/jev2.12089.

Persistent Link:

http://hdl.handle.net/11343/278235

License:

CC BY 